\section{HCV-associated cryoglobulinemia vasculitis: are its days numbered?}

We read with great interest the article by Saadoun et $a l^{1}$ who investigated the efficacy and safety of a 24 -week treatment with sofosbuvir and ribavirin in a small, open-label and uncontrolled study of patients with hepatitis $\mathrm{C}$ virus (HCV)-associated cryoglobulinemia vasculitis. However, the limitations of the VASCUVALDIC study diminish the validity of the conclusions, although we agree with the authors that it would be unethical to delay effective antiviral therapy given the established role of $\mathrm{HCV}$ in the development of cryoglobulinemia vasculitis and its unfavourable prognosis.

As expected, the rate of sustained virological response (SVR) was higher $(74 \%)$ than in previous trials of interferon-based antiviral treatment in patients with $\mathrm{HCV}$-associated cryoglobulinemia vasculitis. ${ }^{2}{ }^{3}$ However, this looks relatively moderate in light of the current efficacy of the interferon-free, all-oral antiviral regimens (up to $95 \%-100 \%$ ). Nonetheless, the SVR rate was comparable with that identified in the previous phase III sofosbuvir plus ribavirin trial in patients with HCV infection. ${ }^{4}$ We can also assume that the proportion of virological responders among patients with HCV-associated cryoglobulinemia vasculitis will increase further due to the use of other available and emerging direct-acting antivirals. Moreover, ribavirin-free regimen may improve the safety of antiviral treatment, given a high rate of anaemia $(25 \%)$ in this study.

Over 24 months, almost $90 \%$ of patients achieved a complete clinical response that was defined by an improvement in all vasculitis manifestations and the absence of clinical relapse. In the majority of responders, the clinical and virological effects were rapid and apparent within the first 12 weeks of treatment. Approximately $30 \%$ of patients received treatment with immunomodulatory or immunosuppressive therapy (including rituximab) that may contribute to, but could not explain, the impressive results of the VASCUVALDIC study. Notably, SVR12 was achieved in all three patients who received a rituximab infusion 1 month prior to the antiviral therapy. Rituximab did not seem to impair the antiviral activity of sofosbuvir plus ribavirin and it can be used concomitantly with direct-acting antivirals. These reassuring findings are important, as antiviral treatment does not eliminate the need for immunosuppression in patients with severe cryoglobulinemia glomerulonephritis or nervous system disease.

The relatively short duration of the follow-up in the current study may have impaired the evaluation of the effect of HCV eradication on the risk of relapse and the need for glucocorticosteroids and immunosuppressants. Previous studies suggest that cryoglobulinemia vasculitis persists or relapses in a small proportion of patients despite a sustained virological response to antiviral treatment. A relapsing course of vasculitis is not related to a persistence of occult viral infection, as transcriptionmediated amplification assays for HCV RNA and tests for HCV RNA replication in peripheral blood mononuclear cells were negative in such patients. ${ }^{5}$ Rarely, B-cell lymphoma can be detected in patients with vasculitis flare although persisting virus-induced immunological abnormalities are a more probable cause for a relapsing course of cryoglobulinemia vasculitis.

The advantages of sofosbuvir and other direct-acting antivirals include a short-duration treatment (usually 12 weeks and up to 8 weeks in selected patients), convenience (as few as one pill a day), a very high response rate and a favourable safety profile with few adverse events. Therefore, direct-acting antivirals have the potential to be the basis for a large expansion in the number of persons treated. ${ }^{6}$ Mathematical models suggest that even a modest increase in treatment coverage can result in a reduction in the HCV prevalence. ${ }^{7}$ According to current estimates, a $90 \%$ reduction in total $\mathrm{HCV}$ infections within 15 years is feasible in many countries studied, but increased capacity for screening and treatment will be critical to achieving this aim. ${ }^{8}$ Wider treatment coverage of patients with HCV infection using very effective and safe direct-acting antivirals may have a profound effect on the epidemiology of $\mathrm{HCV}$-associated cryoglobulinemia vasculitis and other extrahepatic manifestations of HCV infection. Over the last decade, vaccination against HBV infection was associated with a significant decrease in the incidence of polyarteritis nodosa (PAN). In the year 2000, a prevalence of PAN in the French urban multiethnic population exceeded that of granulomatosis with polyangiitis (GPA), microscopic polyangiitis (MPA) and eosinophilic GPA (EGPA). ${ }^{9}$ We lack reliable evidence on the current epidemiology of PAN. However, investigators from 129 centres in Europe, North America and the rest of the world were able to enrol only 142 patients with PAN in the Diagnostic and Classification Criteria in Vasculitis Study versus 901 patients with GPA, 432 patients with MPA and 324 patients with EGPA (as of March 2016). We can expect that a wider treatment for HCV infection may lead to a similar reduction in the prevalence of $\mathrm{HCV}$-associated cryoglobulinemia vasculitis, at least in countries that can afford the high cost of antiviral therapy.

In conclusion, the Saadoun et al's study has important implications for rheumatologists and confirms that treatment for HCV infection should be a priority in patients with $\mathrm{HCV}$-associated cryoglobulinemia vasculitis. The ability of antivirals to cure cryoglobulinemia vasculitis should not be overstated, as a significant proportion of patients (up to one-third in this study) still require immunomodulatory or immunosuppressive therapy to achieve remission. Therefore, rituximab will continue to play an important role in the treatment for HCV-associated cryoglobulinemia vasculitis, particularly where severe kidney or nervous system disease is present.

\section{Sergey Moiseev, Pavel Novikov, Nikolay Mukhin}

Clinic of Nephrology, Internal and Occupational Diseases, Sechenov First Moscow State Medical University, Moscow, Russia

Correspondence to Professor Sergey Moiseev, Clinic of Nephrology, Internal and Occupational Diseases, Sechenov First Moscow State Medical University, Rossolimo, 11/5, Moscow 119435, Russia; clinpharm@mtu-net.ru

\section{Competing interests None.}

Provenance and peer review Not commissioned; internally peer reviewed.

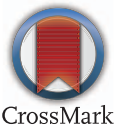

To cite Moiseev S, Novikov P, Mukhin N. Ann Rheum Dis Published Online First: [please include Day Month Year] doi:10.1136/annrheumdis-2016-210636

Accepted 4 October 2016

Ann Rheum Dis 2016:0:1-2. doi:10.1136/annrheumdis-2016-210636

\section{REFERENCES}

1 Saadoun D, Thibault V, Si Ahmed SN, et al. Sofosbuvir plus ribavirin for hepatitis C virus-associated cryoglobulinaemia vasculitis: VASCUVALDIC study. Ann Rheum Dis 2016;75:1777-82.

2 Saadoun D, Resche Rigon M, Thibault V, et al. Peg-IFN $\alpha /$ ribavirin/protease inhibitor combination in hepatitis $C$ virus associated mixed cryoglobulinemia vasculitis: results at week 24. Ann Rheum Dis 2014;73:831-7.

3 Ignatova T, Chernova O, Novikov $\mathrm{P}$, et al. HCV-associated cryoglobulinaemic vasculitis: triple/dual antiviral treatment and/or rituximab? Ann Rheum Dis 2014;73:e58. 
4 Isakov V, Zhdanov K, Kersey K, et al. Efficacy of sofosbuvir plus ribavirin in treatment-naive patients with genotype 1 and 3 HCV infection: results from a Russian Phase Illb study. Antivir Ther 2016. doi:10.3851/IMP3065. [Epub ahead of print 4 Jul 2016].

5 Landau DA, Saadoun D, Halfon P, et al. Relapse of hepatitis C virus-associated mixed cryoglobulinemia vasculitis in patients with sustained viral response. Arthritis Rheum 2008;58:604-11.

6 WHO Guidelines Approved by the Guidelines Review Committee. Guidelines for the screening care and treatment of persons with chronic hepatitis C infection: updated version. Geneva: World Health Organization, 2016.
7 Martin NK, Vickerman P, Grebely J, et al. Hepatitis C virus treatment for prevention among people who inject drugs: modeling treatment scale-up in the age of direct-acting antivirals. Hepatology 2013;58:1598-609.

8 Alfaleh FZ, Nugrahini N, Matičic M, et al. Strategies to manage hepatitis C virus infection disease burden—volume 3. J Viral Hepat 2015;22(Suppl 4):42-65.

9 Mahr A, Guillevin L, Poissonnet M, et al. Prevalences of polyarteritis nodosa, microscopic polyangiitis, Wegener's granulomatosis, and Churg-Strauss syndrome in a French urban multiethnic population in 2000: a capture-recapture estimate. Arthritis Rheum 2004;51:92-9. 